\title{
Theoretical investigation of lattice thermal conductivity and electrophononic effects in $\mathrm{SrTiO}_{3}$
}

\author{
Pol Torres, ${ }^{1, *}$ Juan Antonio Seijas-Bellido, ${ }^{1}$ Carlos Escorihuela-Sayalero, ${ }^{2}$ Jorge Íñiguez, ${ }^{2,3}$ and Riccardo Rurali ${ }^{1}$ \\ ${ }^{1}$ Institut de Ciència de Materials de Barcelona (ICMAB-CSIC), Campus de Bellaterra, E-08193 Bellaterra, Catalonia, Spain \\ ${ }^{2}$ Materials Research and Technology Department, Luxembourg Institute of Science and Technology (LIST), \\ Avenue des Hauts-Fourneaux 5, L-4362 Esch/Alzette, Luxembourg \\ ${ }^{3}$ Physics and Materials Science Research Unit, University of Luxembourg, 41 Rue du Brill, L-4422 Belvaux, Luxembourg
}

(Received 12 November 2018; revised manuscript received 17 February 2019; published 10 April 2019)

\begin{abstract}
We present a theoretical study of the lattice thermal conductivity of $\mathrm{SrTiO}_{3}$ in its antiferrodistortive ferroelastic phase and of its dependence on an applied external electric field, via electrophononic couplings. The calculations are done by using second-principles density-functional theory and the full solution of the Boltzmann transport equation. Our results allow, on one hand, to identify and explain deviations from the usual temperature dependence of the thermal conductivity, revealing Poiseuille flow and a rare umklapp transport regime, in agreement with recent experimental results [Martelli et al., Phys. Rev. Lett. 120, 125901 (2018)]; on the other hand, they show that an external electric field, by reducing the symmetry of the lattice, activates different phonon-phonon scattering processes and thus yields a reduction of the thermal conductivity, supporting the generality of a heat control strategy previously reported by some of us [Seijas-Bellido et al., Phys. Rev. B 97, 184306 (2018)].
\end{abstract}

DOI: 10.1103/PhysRevMaterials.3.044404

\section{INTRODUCTION}

The study of the microscopic factors that govern heat transport, whose ultimate goal is the design of materials with a tailor-made thermal conductivity, is a field of increasing interest $[1,2]$. The thermal conductivity of a material must be high to keep a low thermal budget in a nanoscale device [3], for instance, while it should be as low as possible when it comes to the engineering of an efficient thermoelectric [4,5]. The thermal conductivity can be tuned to some extent by nanostructuring, adding defects [6,7], or designing periodic superstructures [8,9], but it would be desirable to be able to dynamically modify it. Besides an increased flexibility in materials design, this would also open the way to heat-based signal processing and computing [10].

Phonons, the basic quasiparticles that carry heat in a semiconductor or an insulator, are quanta of the collective vibrations of the lattice. Whenever atoms with different nominal charges are present in the crystal, it is possible, at least in principle, to control phonons by an external applied electric field. This approach provides a simple and flexible way to dynamically tune the thermal conductivity of a material, but it is subordinated to the availability of materials with large dielectric responses, i.e., materials where an applied electric field results in a significant distortion of the lattice and the heat carrying phonons are affected in a considerable way. In a previous paper we demonstrated such an electrophononic effect in $\mathrm{PbTiO}_{3}$ (PTO), a ferroelectric oxide where a suitably oriented electric field was shown to be capable of hardening or softening the modes throughout the spectrum or, most

\footnotetext{
*ptorres@icmab.es
}

interestingly for transport-control purposes, to activate different phonon-phonon scattering processes that would be otherwise forbidden by symmetry [11], thus leading to a modulation of the thermal conductivity.

Here, we study, by atomistic theoretical calculations, the heat transport properties of paradigmatic perovskite oxide $\mathrm{SrTiO}_{3}$ (STO). Besides contributing to the basic understanding of the material's behavior, we are interested in assessing to what extent the conductivity can be tuned via the electrophonic effects, previously reported in $\mathrm{PbTiO}_{3}$. At room temperature, STO has a cubic structure. Below $\sim 105 \mathrm{~K}$ it exhibits a soft-mode phase transition to an antiferrodistortive (AFD) ferroelastic phase, where the structure becomes tetragonal and the oxygens of the primitive cell rotate about $2^{\circ}$ degrees in the plane perpendicular to the tetragonal axis [12-15], as shown in the sketch of Fig. 1. Recent theoretical studies using first-principles molecular dynamics and anharmonic lattice dynamics have been able to obtain the phonon band structures including temperature effects and, adopting the relaxation time approximation (RTA), have concluded that optical phonons have a large contribution to the thermal conductivity [16]. In addition, it has been demonstrated experimentally that $\mathrm{SrTiO}_{3}$ can exhibit hydrodynamic effects at low temperature and that the umklapp scattering above $30 \mathrm{~K}$ is affected by AFD fluctuations, showing a trend quite different from that observed in other ferroic perovskites [17,18]. In this paper we study the thermal conductivity of STO in its AFD phase and show that external electric fields allow us to modulate it: The field causes off-center displacements of the cations that thus reduce the symmetry of the crystal lattice; this in turn activates different anharmonic scattering processes that lead to a reduction of the lifetimes of midfrequency phonons that result in an increased resistance to heat currents. 
(a)

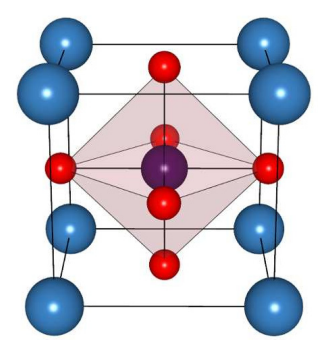

(b)

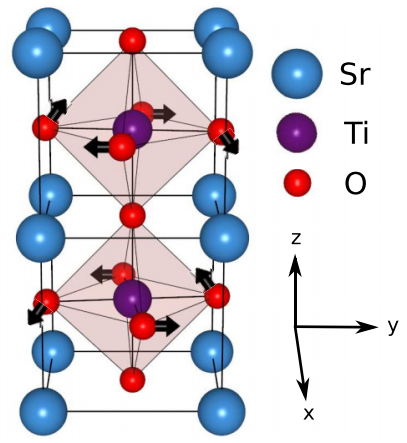

FIG. 1. Sketch of $\mathrm{SrTiO}_{3}$ in (a) the cubic paraelectric and (b) the AFD ferroelastic phase.

\section{COMPUTATIONAL METHODS}

To investigate $\mathrm{SrTiO}_{3}$, we use the second-principles polynomial potential reported in Ref. [15] and labeled "EHM@10K" therein. This model was fitted to reproduce relevant first-principles results with great accuracy. As shown in Ref. [15], the model reproduces the structural phase transition known to occur in $\mathrm{SrTiO}_{3}$, but at a significantly higher temperature than experiment ( 300 vs $105 \mathrm{~K}$ ). Briefly, the reasons for the discrepancy can be traced back to the wellknown tendency of the local density approximation (LDA) to overbind (which can be estimated to account for about $80 \mathrm{~K}$ of the deviation) and the classical treatment of the atoms in regular Monte Carlo simulations (which can be estimated to account for about $70 \mathrm{~K}$ of the deviation [19]). The tilt of the $\mathrm{O}_{6}$ groups is also exaggerated by the model as compared to experiment ( $6.5^{\circ}$ vs $2^{\circ}$, approximately). This large value is LDA's prediction, which our model captures very accurately; hybrid functionals [20] have been shown to considerably improve the description.

Then, we run all our calculations (structural relaxations, calculation of interatomic force constants under applied electric fields, etc.) using standard methods implemented in the SCALE UP code $[14,21]$. In order to perform these calculations we use a cell of 40 atoms, which can be viewed as a $2 \times 2 \times 2$ repetition of the 5-atom elemental perovskite unit cell. This large cell allows us to describe properly the AFD phase as well as other phases combining AFD and polar distortions that might appear as a result of the electric field.

We use the PHONOPY [22] and PHONO3PY [23] codes to create supercells with all the inequivalent displacement needed to compute the harmonic and anharmonic force constants of the crystal. For that we use the finite-difference method in a $2 \times 2 \times 2$ supercell of the previous one, i.e., a 320-atom cell, without any limitation in the range of the interactions. Once the supercells are generated the forces are calculated within second-principles density functional theory (DFT) using SCALE UP. PHONOPY is also used to plot the phonon dispersion from the harmonic force constants. The reported dielectric susceptibility corresponds to the dominant lattice part, and is also obtained from our second-principles model from finite-field calculations.

The thermal conductivity for the different configurations, with and without an external field, is calculated through the direct solution of the linearized phonon Boltzmann transport

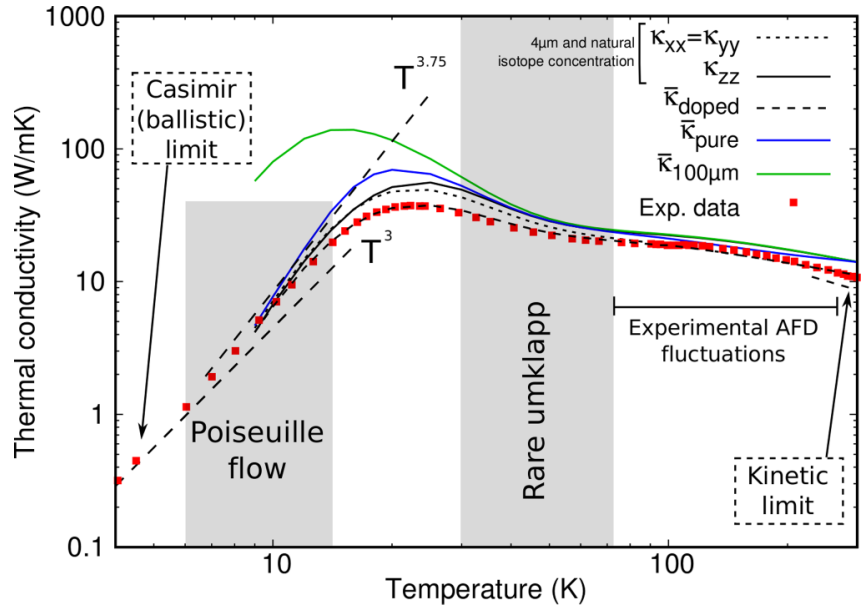

FIG. 2. Thermal conductivity of $\mathrm{SrTiO}_{3}$ in the AFD phase as a function of temperature. The faster-than-cubic temperature dependence of $\boldsymbol{\kappa}$ indicates a Poiseuille transport regime where normal processes dominate aharmonic scattering. The $T^{3}$ dependence expected when phonon transport is limited by boundary scattering is shown for comparison. The thermal conductivity of an isotopically purified sample is shown with a blue solid line $\left(\bar{\kappa}_{\text {pure }}\right)$, while $\bar{\kappa}_{\text {doped }}$ corresponds to the case of a lanthanum-doped system, $\mathrm{Sr}_{1-x} \mathrm{La}_{x} \mathrm{TiO}_{3}$, with $x=0.00085$. A calculation with a boundary scattering of $100 \mu \mathrm{m}\left(\bar{\kappa}_{100 \mu \mathrm{m}}\right)$ is also included to observe the size effects at low temperature. Experimental data (red squares) are taken from Ref. [18].

equation (LBTE) [24] as implemented in the PHONO3PY code. The mode-specific thermal conductivity is given by

$$
\kappa^{\alpha \beta}(\omega)=\int \frac{\rho_{\alpha \beta}\left(\omega^{\prime}\right)}{\omega^{\prime}-i \omega} d \omega^{\prime},
$$

where $\rho_{\alpha \beta}\left(\omega^{\prime}\right)$ is the spectral density [24]. We solve Eq. (1) on an $8 \times 8 \times 8 \mathbf{q}$-point grid and obtain the thermal conductivity $\boldsymbol{\kappa}(T)$ by summing over all the modes. We have also included isotopic disorder scattering, using the natural abundances of isotopes of $\mathrm{Sr}, \mathrm{Ti}$, and $\mathrm{O}$, within the model of Tamura [25]. Let us note that, within the LBTE approximation, the temperature only enters via the phonon populations. Such an approach cannot capture the temperature-driven phase transition occurring in $\mathrm{SrTiO}_{3}$; thus, we expect the LBTE results to be relevant at temperatures below the transition, and not strictly reliable at temperatures above.

\section{RESULTS AND DISCUSSION}

We start our study by calculating the thermal conductivity of $\mathrm{SrTiO}_{3}$ in its low-temperature AFD phase and in the absence of an external electric field. The results are shown in Fig. 2. As we can observe, the thermal conductivity features a small anisotropy, with $\kappa_{z z}>\kappa_{x x}=\kappa_{y y}$, as expected from the symmetry of the lattice, and has a peak at around $20 \mathrm{~K}$, before umklapp scattering (i.e., phonon-phonon collisions that do not conserve momentum) starts to be the dominant scattering mechanism. The calculated results are in good agreement with the recent experimental report by Martelli and co-workers [18], who measured the thermal conductivity of STO from 2 to $400 \mathrm{~K}$. This fairly good agreement is especially remarkable 
given the anomalous features of $\boldsymbol{\kappa}(T)$ highlighted in the experiments, such as a Poiseuille phonon flow, a heat transport regime where momentum conserving phonon-phonon collisions (normal scattering) result in a faster-than-cubic increase of $\boldsymbol{\kappa}(T)$ below its peak $\left(\boldsymbol{\kappa} \propto T^{3.75}\right.$ in our calculations). It is important to recall that our results rely on force constants calculated in the AFD ground state, and thus no phase transition can be accounted for. Indeed, the computed and measured thermal conductivities move away from each other at around $100 \mathrm{~K}$, which is the temperature where the experimental sample stops being tetragonal and becomes cubic. For the same reason we suggest that the heat transport regime dominated by rare umklapp scattering events extends to higher temperatures than those proposed in Ref. [18]. The strong AFD fluctuations-i.e., oxygen rotations associated to the AFD transition-that dominate up to room temperature are not properly captured by our approach, and thus the good agreement up to $\sim 100 \mathrm{~K}$ indicates that they are activated above the phase transition. The somewhat less satisfactory agreement in the $20-40 \mathrm{~K}$ temperature range might be due to impurities in the experimental sample that are not accounted for by just including the isotopic disorder of natural abundance. We have verified, indeed, that by including a lanthanum (La) impurity concentration of $x=0.00085\left(\mathrm{Sr}_{1-x} \mathrm{La}_{x} \mathrm{TiO}_{3}\right)$, we can recover the experimental data ( $\bar{\kappa}_{\text {doped }}$ in Fig. 2); in order to account for these impurities we change the mass variance term from Tamura's expression [25] according to the mentioned concentration. For the very-low-temperature regime we have used a boundary scattering according to the Casimir limit (i.e., the ballistic limit for a finite-size model) corresponding to a sample or grain size of $4 \mu \mathrm{m}$ to reproduce the real size effects of the experimental data. We notice, however, that we were not able to converge the solution of the LBTE below $9 \mathrm{~K}$. In Fig. 2 we also plot for comparison the thermal conductivity with a boundary scattering term corresponding to a sample of $100 \mu \mathrm{m}\left(\bar{\kappa}_{100 \mu \mathrm{m}}\right)$, such as the one used in Ref. [26], and a sample with an isotopically pure concentration $\left(\bar{\kappa}_{\text {pure }}\right)$. As expected, the results are different at low temperatures, where boundary and isotope scattering is important, and tend to become indistinguishable at higher temperatures.

We now move to the study of electrophononic effects, i.e., the dependence of the thermal conductivity on external electric fields. Before that, nonetheless, we calculate the polarization as a function of the electric field within second-principles DFT, $\mathbf{P}(\mathbf{E})$, and extract the lattice electrical susceptibility $\chi$ from $\mathbf{P}=\epsilon_{0} \chi \mathbf{E}$, where $\epsilon_{0}$ is the electrical permittivity in vacuum. Notice that $\chi$ plays a crucial role in the estimate of the variation of $\boldsymbol{\kappa}$ in the presence of an applied electric field: The value of $\boldsymbol{\chi}$ is an indication of how much the atoms are displaced from their equilibrium positions when a field is applied, and large displacements are expected to lead to large variations of $\boldsymbol{\kappa}$. We obtained 426 and 234 for $\chi_{x x}=\chi_{y y}$ and $\chi_{z z}$, respectively. These values suggest that STO can exhibit electrophononic effects similar to those recently reported for $\mathrm{PbTiO}_{3}$ that have $\chi_{x x}=\chi_{y y}=304$ and $\chi_{z z}=31$ [17]. At very low temperatures, $\mathrm{SrTiO}_{3}$ is known to enter a so-called quantum paraelectric regime characterized by much larger dielectric responses [27]. This is not captured by our present simulations, which underestimate the response to electric fields at low temperatures. Therefore, the magnitude of the

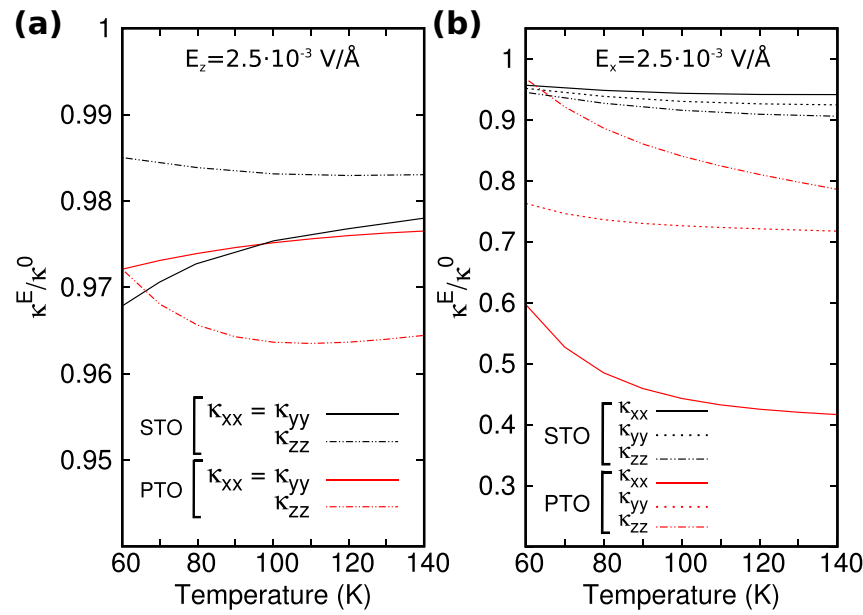

FIG. 3. Variation of the thermal conductivity as a function of temperature for (a) $E_{z}=2.5 \times 10^{-3} \mathrm{~V} / \AA$ and (b) $E_{x}=2.5 \times 10^{-3}$ $\mathrm{V} / \AA$. The changes are referred to the value of the thermal conductivity $\boldsymbol{\kappa}^{0}$ without field. For comparison the variation of the thermal conductivity of PTO with an electric field of the same value opposed to the polarization $\left(-E_{z}\right)$ is shown in (a) and with the same value $\left(E_{x}\right)$ in (b) [17].

computed electrophononic effects can be considered the lower limit of the effect that one would measure experimentally.

We calculated the thermal conductivity in the presence of external fields $E_{z}\left(E_{x}\right)$ parallel (perpendicular) to the tetragonal axis and with values up to $4.5 \times 10^{-3} \mathrm{~V} / \AA$. The relative changes of the diagonal components of the thermal conductivity tensor $\kappa_{x x}, \kappa_{y y}$, and $\kappa_{z z}$, for the same values of $E_{z}$ and $E_{x}$, are plotted in Fig. 3. As we can see there, the reduction of $\boldsymbol{\kappa}$ is larger for fields perpendicular to the tetragonal axis $E_{x}$. In addition, we notice that the trend in temperature is different depending on the direction of the field. While for parallel fields $\boldsymbol{\kappa}$ decreases when reducing $T$, it increases for the perpendicular case.

To better characterize the dependence of the thermal conductivity on the electric field, we have calculated $\boldsymbol{\kappa}$ on a finer grid of values of $E_{z}$ and $E_{x}$ at $100 \mathrm{~K}$, as shown in Fig. 4. From
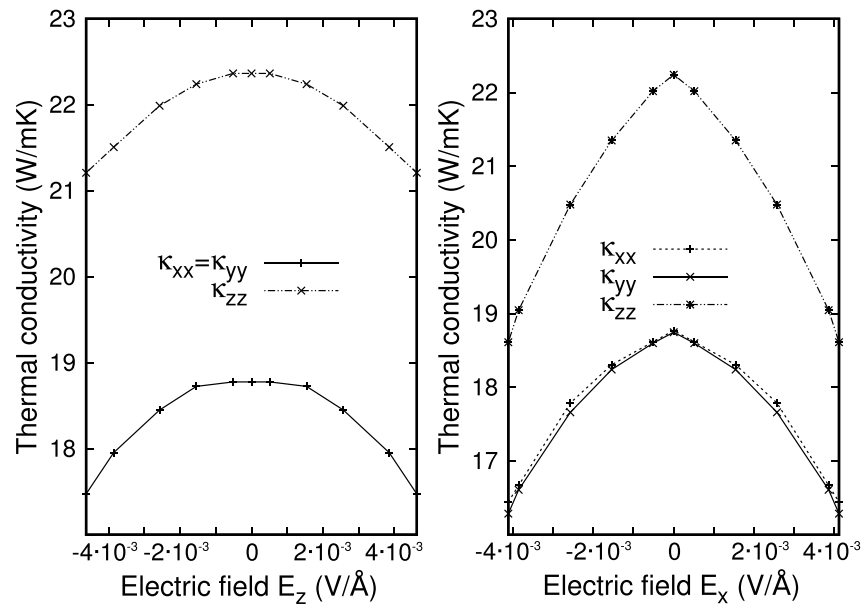

FIG. 4. Thermal conductivity as a function of an external electric field $E_{z}$ (left) and $E_{x}$ (right) at $T=100 \mathrm{~K}$. 


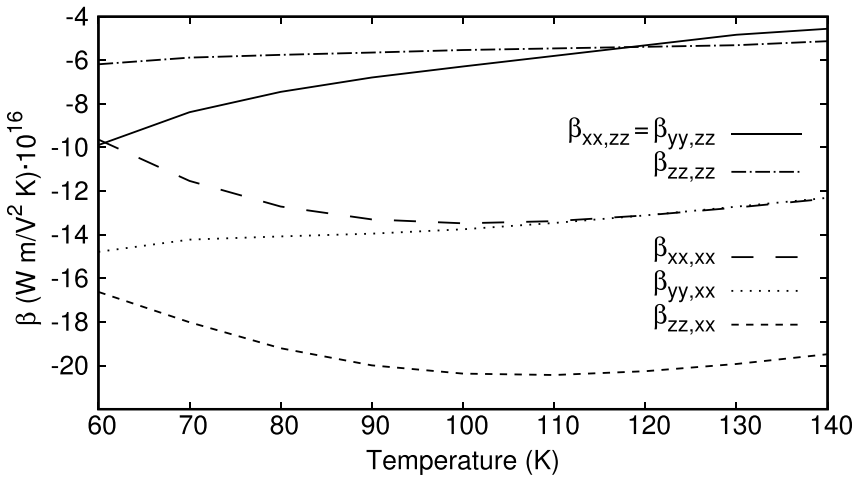

FIG. 5. Diagonal components of the second-order electrophononic tensor $\boldsymbol{\beta}_{i j, k l}$, as defined in Eq. (2), as a function of temperature.

this figure, we observe that all the components of the thermal conductivity are a decreasing function of the electric field. While the behaviors of $\kappa_{x x}$ and $\kappa_{y y}$ are very similar for both directions of the electric field, $\kappa_{z z}$ exhibits a stronger response in the case of $E_{x}$. The results in Fig. 4 can be fitted to

$$
\kappa_{i j}(T, \mathbf{E})=\kappa_{i j}^{0}(T)+\sum_{k} \alpha_{i j, k}(T) E_{k}+\sum_{k l} \beta_{i j, k l}(T) E_{k} E_{l},
$$

where $\boldsymbol{\alpha}$ and $\boldsymbol{\beta}$ are the electrophononic tensors and $\boldsymbol{\kappa}^{0}$ is the conductivity at zero field. In our case, all the components of the linear tensor $\alpha$ are zero by symmetry, which we confirmed numerically. Then, the calculated values of the thermal conductivity can be nicely fitted with a quadratic dependence on the electric field. The temperature dependence of the quadratic electrophononic tensor $\boldsymbol{\beta}_{i j, k l}$ is plotted in Fig. 5. Here, one can see that the reduction induced by a given electric field $E_{z}$ is stronger at low temperature, i.e., the absolute value of $\beta_{i i, z z}$ is larger, although the highest reduction is found for a perpendicular field. The quadratic dependence of $\boldsymbol{\kappa}$ on the electric field allows rationalizing the results reported in Fig. 3, where the relative variation of the thermal conductivity of PTO in response to an antiparallel and perpendicular field $[11,17]$ is also reported for comparison. The electrophononic response of PTO for this kind of applied field is linear because of the symmetry breaking associated with the ferroelectric distortion in that material and the reduction of $\boldsymbol{\kappa}$ in the limit of low fields is larger than the one in STO [17]. When the field increases, however, $\boldsymbol{\kappa}$ decreases faster in the case of STO because of its quadratic dependence on the applied field and thus the reduction can eventually be larger than in PTO. In spite of this, the largest reductions are still those observed in response to an electric field perpendicular to the polar axis of PTO, which forces the rotation of the polarization [17].

Having a look at the phonon bands, in Fig. 6(a) we observe that the electric field induces a reduction in frequency of the acoustic modes. In contrast, optical modes do not follow a specific pattern in this respect and we do not observe a general softening of all the modes as in the case of fields opposed to the polarization in $\mathrm{PbTiO}_{3}$ (see Ref. [17]). From Fig. 6(b) we observe a general increase in the scattering matrix elements $|V(\omega)|^{2}$ (i.e., phonon collision strength calculated from the
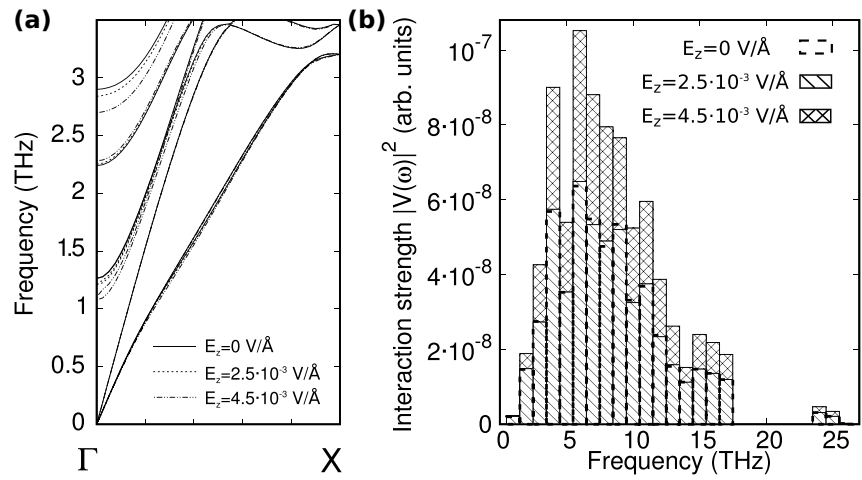

FIG. 6. (a) Phonon band structure of $\mathrm{SrTiO}_{3}$ along the $\Gamma-X$ direction. (b) Phonon-phonon scattering interaction strength for $\mathrm{SrTiO}_{3}$ with $E_{z}=0 \mathrm{~V} / \AA, E_{z}=2.5 \times 10^{-3} \mathrm{~V} / \AA$, and $E_{z}=4.5 \times 10^{-3} \mathrm{~V} / \AA$ at $100 \mathrm{~K}$.

interatomic force constants [28]) as the electric field increases. These results reflect the fact that, as previously found in the case of the response of $\mathrm{PbTiO}_{3}$ to fields perpendicular to the polarization [17], the applied electric field reduces the symmetry of the lattice, activating more three-phonon scattering processes, which in turn yields larger $|V(\omega)|^{2}$. The enhanced scattering results in shorter phonon relaxation times, being therefore mainly responsible for the smaller thermal conductivity. In addition, we notice that the higher values of $|V(\omega)|^{2}$ correspond to midfrequency optical phonons.

To further dig into the origins of the reduction of the thermal conductivity with an external electric field and elucidate which one of the two effects reported in Figs. 6(a) and 6(b) is dominant, we have calculated the participation ratio of each mode to the thermal conductivity. In particular, in Fig. 7 we plot the thermal conductivity accumulation function, defined as $\boldsymbol{\kappa}_{\text {accum }}(\omega)=\int_{0}^{\omega} \boldsymbol{\kappa}\left(\omega^{\prime}\right) d \omega^{\prime}$.

As we can see, the curves with and without field run parallel up to 3-5 $\mathrm{THz}$ where a first decrease appear. Other significant decreases of $\boldsymbol{\kappa}$ in the presence of an applied field show up between 5 and $10 \mathrm{THz}$. Therefore, these data

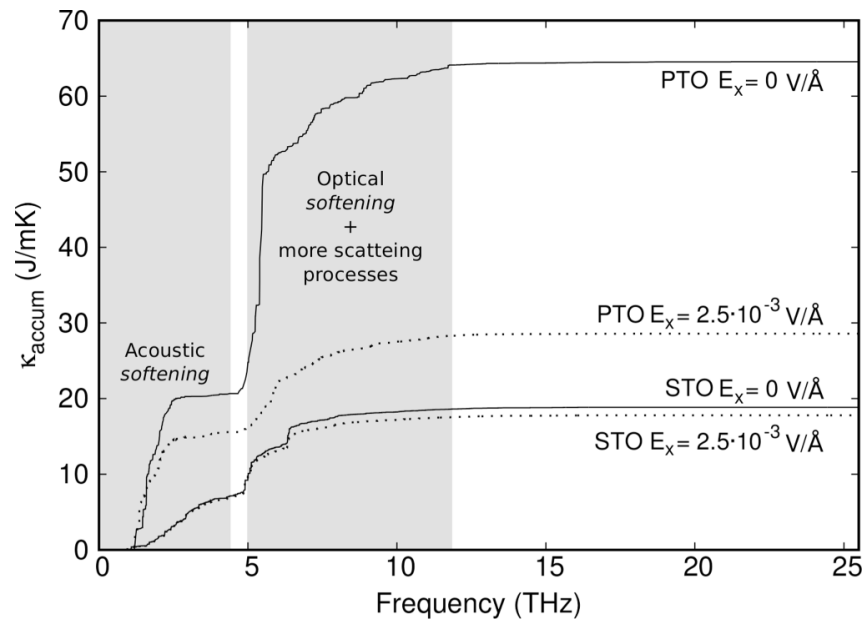

FIG. 7. Thermal conductivity accumulation function for $\mathrm{SrTiO}_{3}$ with $E_{x}=0 \mathrm{~V} / \AA$ and $E_{x}=2.5 \times 10^{-3} \mathrm{~V} / \AA$ at $100 \mathrm{~K}$. Results for $\mathrm{PbTiO}_{3}$ are shown for comparison. 
indicate that the observed softening of the acoustic modes has little effect on the thermal conductivity; instead, its decrease appears to be almost entirely determined by the activation of different anharmonic scattering processes allowed by the symmetry breaking that the electric field causes. In the case of PTO, whose thermal conductivity accumulation function is also shown for comparison in Fig. 7, in addition to the field-induced symmetry break, a global softening of all modes produces a further reduction of $\boldsymbol{\kappa}$ [17]. We also notice that for STO, optical phonons with a frequency higher than $3 \mathrm{THz}$ have an important contribution to the thermal transport, as it has been already reported for STO in the absence of field by Feng et al. [16]. Additionally, we have verified that while the reduction of the thermal conductivity when we apply fields parallel to the tetragonal axis $\left(E_{z}\right)$ is caused by an increase of the interatomic force constants (IFCs), when applying a perpendicular field $\left(E_{x}\right)$, an increase of the three-phonon scattering phase space produces a further reduction in $\kappa$, as observed in Fig. 4.

As a final comment, we observe that our model reproduces well the susceptibility of DFT calculations, which in turn gives a good estimate of the room-temperature experimental value. However, because of the quantum-paraelectric effects mentioned above, experimental results show that $\chi$ experiences a large increase when temperature decreases below $40-50 \mathrm{~K}$ [27]. Thus our predictions should be understood as a lower bound of the actual electrophononic response of STO at low temperatures.

\section{CONCLUSIONS}

We have used second-principles density-functional theory coupled to a full solution of the Boltzmann transport equation to calculate the thermal conductivity of the antiferrodistortive ferroelastic phase of $\mathrm{SrTiO}_{3}$. Our results agree well with recent experimental measurements and they also capture the Poiseuille transport regime observed at low temperature where normal scattering processes are the dominant anharmonic scattering mechanisms. We also studied electrophononic effects and reported a quadratic response of the thermal conductivity to an external applied electric field. Similarly to what was previously reported for $\mathrm{PbTiO}_{3}$, the field-induced reduction of the symmetry of the lattice results in the activation of different three-phonon scattering processes that cause a significant decrease of the thermal conductivity. However, although the electric susceptibility of $\mathrm{SrTiO}_{3}$ is higher, the overall reduction of $\boldsymbol{\kappa}$ is in general larger in the case of $\mathrm{PbTiO}_{3}$ due to a global band softening caused by the field in this material. Yet, the response of the thermal conductivity, according to the experimental electrical susceptibility, can be significant at low temperatures and for specific field configurations. Together with the lack of toxicity of $\mathrm{Sr}$, at variance with $\mathrm{Pb}$, this makes $\mathrm{SrTiO}_{3}$ a good candidate to explore new electrophononic applications.

\section{ACKNOWLEDGMENTS}

We acknowledge financial support by the Ministerio de Economía, Industria y Competitividad (MINECO) under Grants No. FEDER-MAT2013-40581-P and No. FEDERMAT2017-90024-P, the Severo Ochoa Centres of Excellence Program under Grant No. SEV-2015-0496, and by the Generalitat de Catalunya under Grant No. 2017 SGR 1506. Work in Luxembourg was funded by the Luxembourg National Research Fund through the CORE (Grant No. FNR/C15/MS/10458889 NEWALLS, JI) and AFR (Grant No. 9933418, CES) programs.
[1] D. G. Cahill, W. K. Ford, K. E. Goodson, G. D. Mahan, A. Majumdar, H. J. Maris, R. Merlin, and S. R. Phillpot, J. Appl. Phys. 93, 793 (2003).

[2] D. G. Cahill, P. V. Braun, G. Chen, D. R. Clarke, S. Fan, K. E. Goodson, P. Keblinski, W. P. King, G. D. Mahan, A. Majumdar, H. J. Maris, S. R. Phillpot, E. Pop, and L. Shi, Appl. Phys. Rev. 1, 011305 (2014).

[3] A. L. Moore and L. Shi, Mater. Today 17, 163 (2014).

[4] M. Zebarjadi, K. Esfarjani, M. S. Dresselhaus, Z. F. Ren, and G. Chen, Energy Environ. Sci. 5, 5147 (2012).

[5] G. Benenti, G. Casati, K. Saito, and R. S. Whitney, Phys. Rep. 694, 1 (2017).

[6] M. Royo and R. Rurali, Phys. Chem. Chem. Phys. 18, 26262 (2016).

[7] M. B. Bebek, C. M. Stanley, T. M. Gibbons, and S. K. Estreicher, Sci. Rep. 6, 32150 (2016).

[8] M. N. Luckyanova, J. Garg, K. Esfarjani, A. Jandl, M. T. Bulsara, A. J. Schmidt, A. J. Minnich, S. Chen, M. S. Dresselhaus, Z. Ren, E. A. Fitzgerald, and G. Chen, Science 338, 936 (2012).

[9] J. Ravichandran, A. K. Yadav, R. Cheaito, P. B. Rossen, A. Soukiassian, S. J. Suresha, J. C. Duda, B. M. Foley, C.-H. Lee, Y. Zhu, A. W. Lichtenberger, J. E. Moore, D. A. Muller,
D. G. Schlom, P. E. Hopkins, A. Majumdar, R. Ramesh, and M. A. Zurbuchen, Nat. Mater. 13, 168 (2013).

[10] N. Li, J. Ren, L. Wang, G. Zhang, P. Hänggi, and B. Li, Rev. Mod. Phys. 84, 1045 (2012).

[11] J. A. Seijas-Bellido, C. Escorihuela-Sayalero, M. Royo, M. P. Ljungberg, J. C. Wojdel, J. Íñiguez, and R. Rurali, Phys. Rev. B 96, 140101(R) (2017).

[12] J. Sidoruk, J. Leist, H. Gibhardt, M. Meven, K. Hradil, and G. Eckold, J. Phys.: Condens. Matter 22, 235903 (2010).

[13] R. Ouillon, J.-P. Pinan-Lucarre, P. Ranson, P. Pruzan, S.-K. Mishra, R. Ranjan, and D. Pandey, J. Phys.: Condens. Matter 14, 2079 (2002).

[14] J. Wojdel, P. Hermet, M. Ljungberg, P. Ghosez, and J. Íñiguez, J. Phys.: Condens. Matter 25, 305401 (2013).

[15] C. Escorihuela-Sayalero, J. C. Wojdel, and J. Íñiguez, Phys. Rev. B 95, 094115 (2017).

[16] L. Feng, T. Shiga, and J. Shiomi, Appl. Phys. Express 8, 071501 (2015).

[17] J. A. Seijas-Bellido, H. Aramberri, J. Íñiguez, and R. Rurali, Phys. Rev. B 97, 184306 (2018).

[18] V. Martelli, J. L. Jiménez, M. Continentino, E. BaggioSaitovitch, and K. Behni, Phys. Rev. Lett. 120, 125901 (2018).

[19] W. Zhong and D. Vanderbilt, Phys. Rev. B 53, 5047 (1996). 
[20] R. Wahl, D. Vogtenhuber, and G. Kresse, Phys. Rev. B 78, 104116 (2008).

[21] P. García-Fernández, J. C. Wojdeł, J. Íñiguez, and J. Junquera, Phys. Rev. B 93, 195137 (2016); https://www.secondprinciples. unican.es/

[22] A. Togo and I. Tanaka, Scr. Mater. 108, 1 (2015).

[23] A. Togo, L. Chaput, and I. Tanaka, Phys. Rev. B 91, 094306 (2015).
[24] L. Chaput, Phys. Rev. Lett. 110, 265506 (2013).

[25] S. I. Tamura, Phys. Rev. B 27, 858 (1983).

[26] A. Buckley, J. P. Rivera, and E. K. H. Salje, J. Appl. Phys. 86, 1653 (1999).

[27] K. A. Müller and H. Burkard, Phys. Rev. B 19, 3593 (1979).

[28] P. Torres, Thermal Transport in Semiconductors: First Principles and Phonon Hydrodynamics (Springer, Cham, Switzerland, 2018). 